\title{
p63 signaling in BRAF inhibitor resistant melanoma
}

\section{A.Patel $^{1}$, C. Harwood ${ }^{1}$ and D.Bergamaschi ${ }^{1}$}

${ }^{1}$ Centre for Cell Biology and Cutaneous Research, Blizard Institute, Queen Mary University of London, London (UK)

\section{Introduction}

$>$ In cutaneous melanoma around $50 \%$ to $60 \%$ patients carry an activating mutation in BRAF;

> Targeted drugs (BRAF and MEK inhibitors) induced responses in most patients, but nearly all of them relapsed due to pre-existing or acquired resistance;

Over the past few years several mechanisms/pathways have been linked to the resistance in these patients, including acquired mutations in N-RAS and MEK1, increased expression of MAP3K, Cot1, cyclin D1 and several growth factor receptors;

p63 is often expressed in melanoma (50-60\% regardless the stage of the disease) and it is linked with chemoresistance. p63 prevents p53 nuclear stabilization and hence inhibits p53 pro-apoptotic signaling in melanoma;

Figure 1. Resistance mechanisms involved in melanoma
A

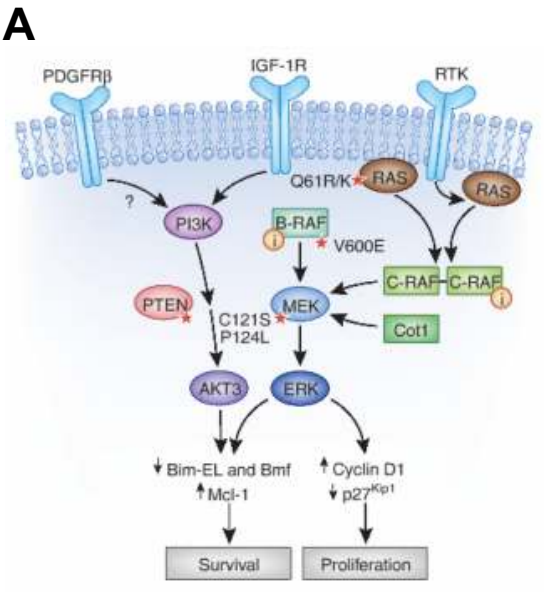

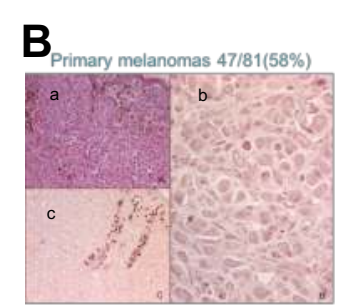

D

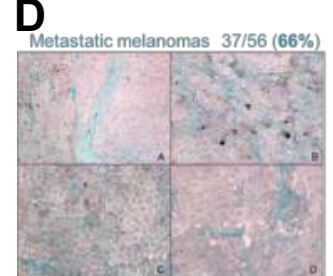

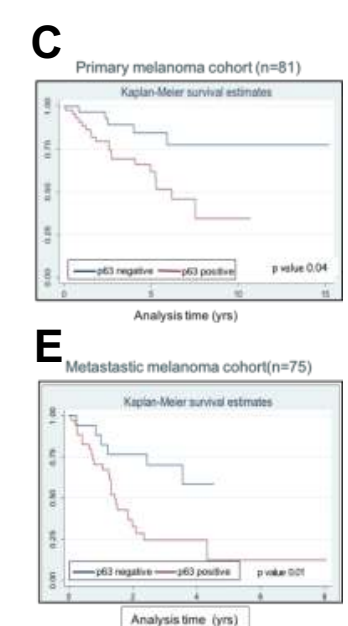
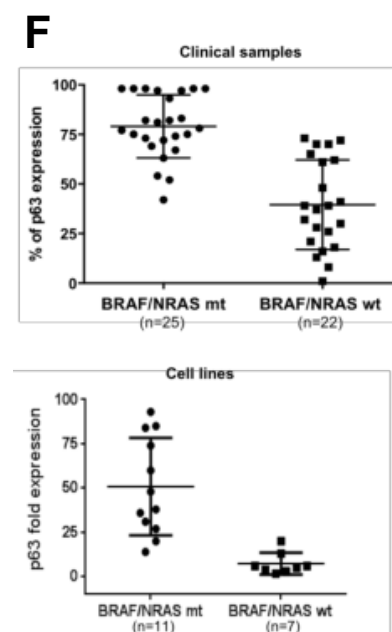

A. Multiple mechanisms of BRAFi resistance in melanoma (Figure adapted from Aplin et. al. 2011).

B. Immunohistochemistry of primary melanoma: (a) Hematoxylin and eosin staining. (b) Higher magnification of melanoma cells showing nuclear
and cytoplasmic p63 reactivity (c) Anti-p63 antibody demonstrating strong nuclear labeling of epidermal keratinocytes confirming positive internal control (Matin et. al. 2013).

C. Kaplan-Meier survival estimates (melanoma specific) in the primary melanoma cohort $(n=81)$ demonstrate p63 reactivity to be a significant D. p63 reactivity in three different lymph nodes completely replaced with metastatic melanoma in a 81 -yr-old male (Matin et. al. 2013).
E. Kaplan-Meier survival estimates (melanoma specific) in the metastatic melanoma cohort $(n=56)$ showing p63 reactivity to be a significant predictor of poorer outcomes (multivariate analysis $\mathrm{HR}$ 3.86; $\mathrm{P}=0.01$, Cox proportional HR)
F. Significant statistical correlation between al. 2013 )
p63 expression and mutational status of BRAF/NRAS in melanoma clinical samples.

Figure 2. Development of BRAFi resistance model in vitro and p63 expression in resistant cells

A

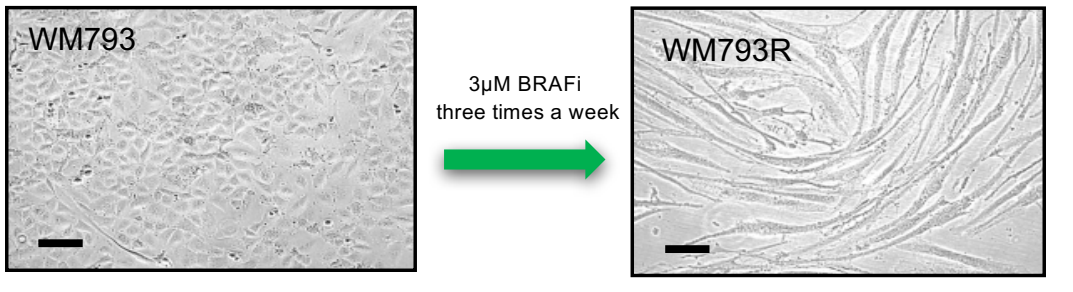

B

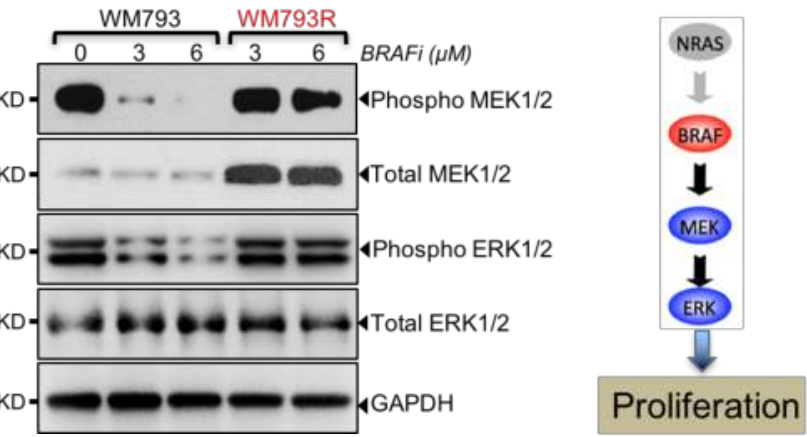

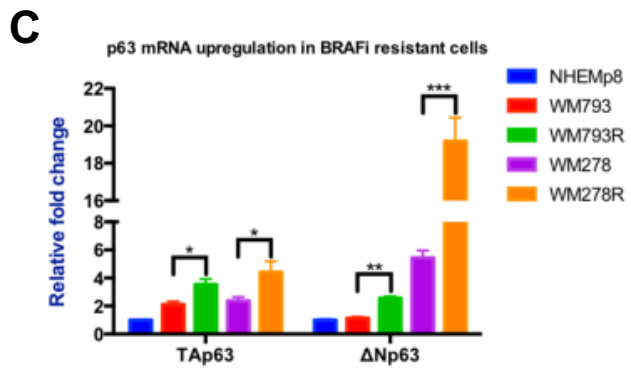

D

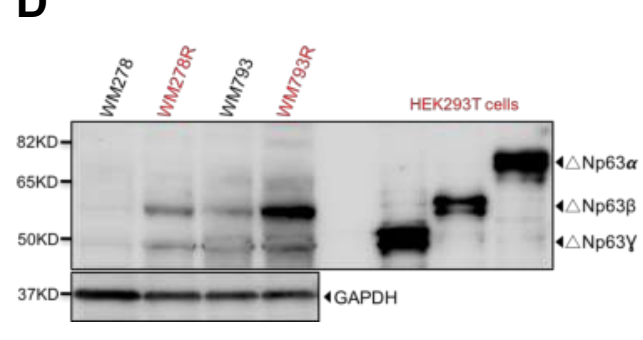

A. WM793 melanoma cells treated with $3 \mu \mathrm{M}$ BRAFi for three times a week, showing phenotypical changes upon acquisition of resistance to the A.w

B. Western Blotting analysis showing the level of MEK $1 / 2$ and ERK $1 / 2$ in parental and resistant cells with or without BRAFi treatment. ${ }^{*}$ Scale bars

$=50 \mu \mathrm{m}$

C. $q R T-P C R$ showing $p 63$ mRNA upregulation in resistant melanoma cells compared to parental cells. Error bars indicate standard error mean.
$* * P<0.01 * * * P<0.001$

D. Western Blot showing upregulation of P63 isoforms in BRAFi resistant cells. GAPDH confirmed equal loading for all proteins.

Figure 3. FBXW7 regulates p63 stability in BRAFi resistant cells

A
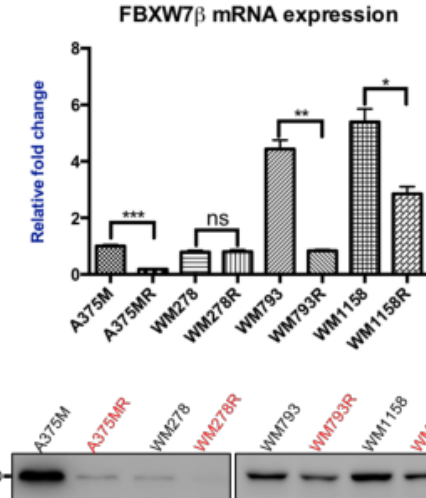

$-$
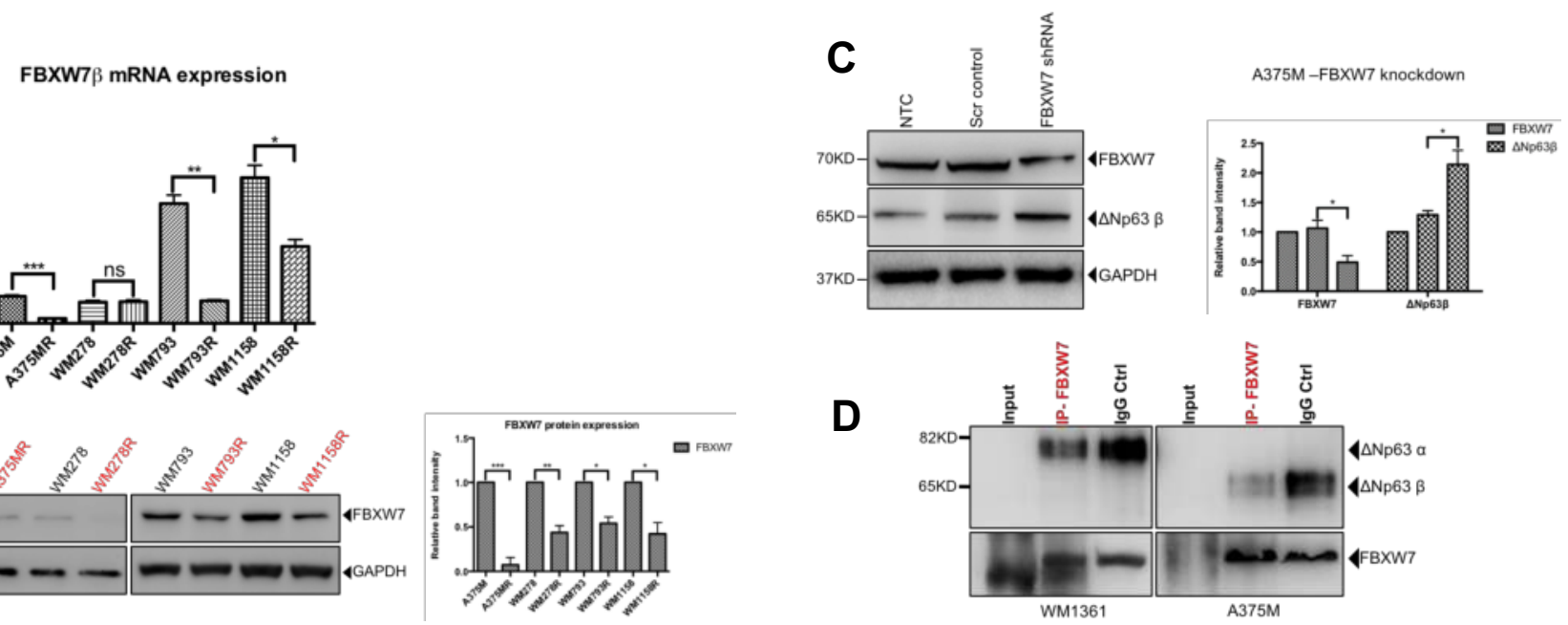

A. gRT-PCR showing downregulatio
normalized to GUS reference gene.

B. Western Blot analysis showing decreased FBXW7 protein level in BRAFi

represents the average band intensity from three independent experiments.

C. shRNA experiments showing increased expression of p63 upon FBXW7 silencing in A375 parental melanoma cell line. Band intensity for
FBXW7 and $\triangle N$ Np633 normalized to GAPDH expression. Error bars indicate standard error mean. ${ }^{*} P<0.05,{ }^{* *} P<0.01$, ${ }^{* * *} P<0.001$, ns FBXW7 and $\triangle$ nonsignificant.
notis

\section{Aims of this study}

To examine the association between p63 expression and BRAF/NRAS mutation in melanoma clinical samples

$>$ To investigate p63 involvement in development of BRAFi-resistance in melanoma

$>$ To characterize potential regulatory mechanisms of p63 in BRAFi-resistant melanoma cells

Figure 4. MDM2 increases in the nucleus in response to Vemurafenib treatment
A
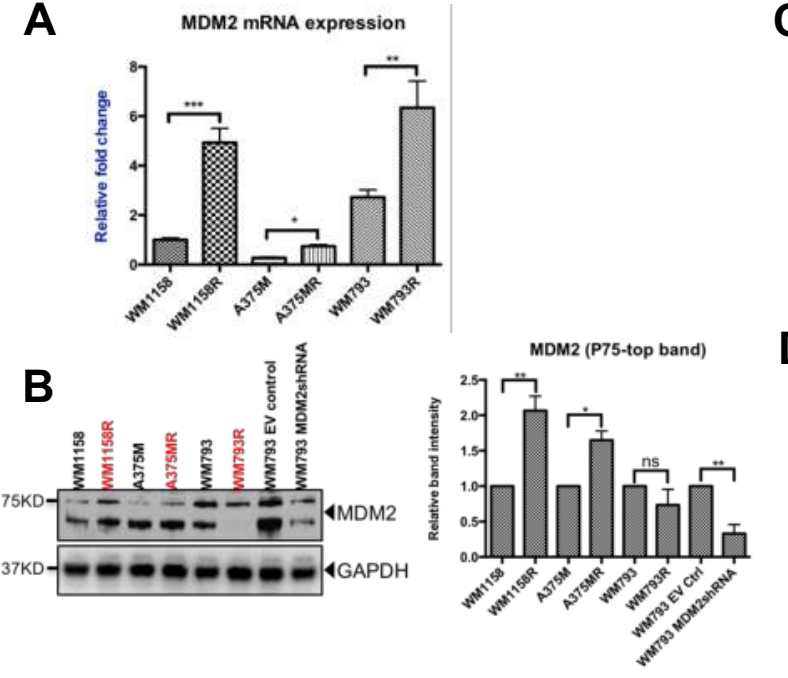

A. qRT-PCR of MDM2 expression in parental and resistant
cells. GUS was used as normalizer for qRT-PCR experiments.

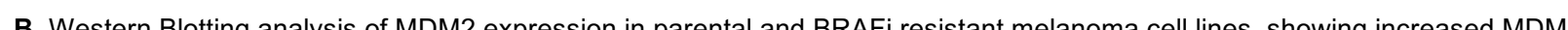
expression in resistant cells. GAPDH confirmed equal loading for all proteins.

C and D. Immunofluorescence and Western blot analysis of MDM2 showing its predominant nuclear localization in response to vemurafenib treatment.

Figure 5. MDM2 regulates FBXW7 stability

A

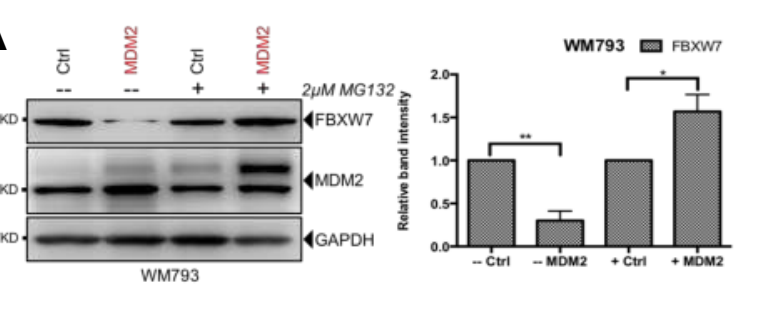

D

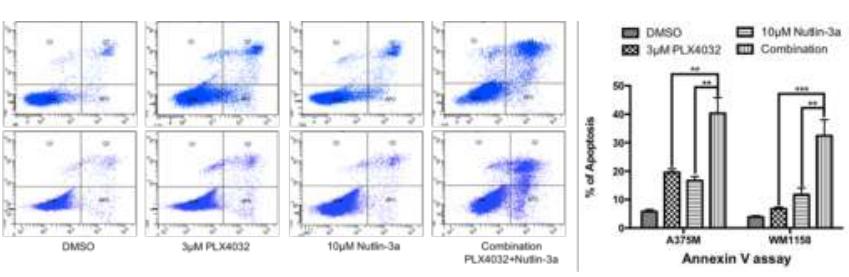

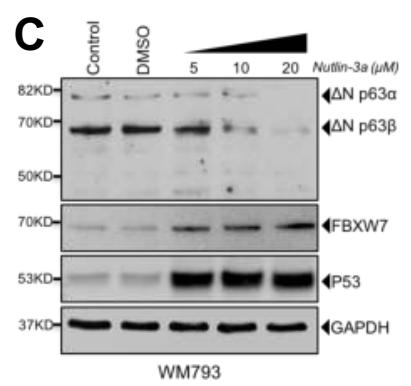

A. Western Blotting analysis of FBXW7 with or without proteasomal in
absence of MG132. GAPDH confirmed equal loading for all proteins.

B. Immunoprecipitation analysis showing direct interaction between MDM2 and FBXW7 in WM793 melanoma cell line. HaCat cells used as positive control and IgG antibody was used as negative internal control. Input represents the total cell lysate.

C. Western blotting analysis showing an inverse correlation in the expression of p63 and FBXW7 upon the addition of increasing
doses of Nutlin-3a drug. GAPDH confirmed equal loading for all proteins.

D. FACS analysis with AnnexinV showing increased apoptosis in melanoma cells after BRAFi and Nutlin-3a combination treatment.

Figure 6. Multiple mechanisms of resistance in melanoma

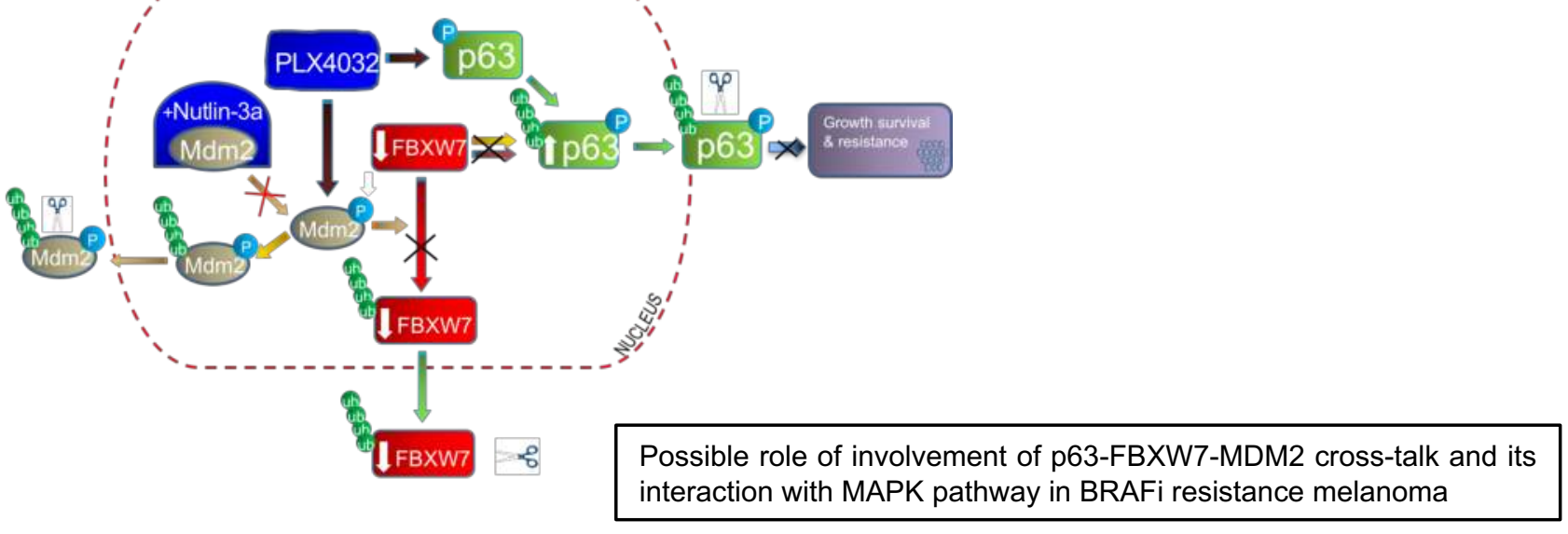

Conclusions

* p63 expression correlates with mutated BRAF/NRAS status in melanoma clinical samples

* p63 is significantly increased at RNA and protein level and confers resistance to apoptosis upon treatment with BRAFi

* BRAFi treatment in melanoma cells lead to FBXW7 and MDM2 accumulation into the nucleus where MDM2 could degrade FBXW7 and may contribute to p63 protein stabilization

* Combined treatment of BRAFi with Nutlin-3a could be more effective in inducing apoptosis in melanoma cells then BRAFi treatment alone 